\title{
Protective Effects of p38 MAPK Inhibitor SB202190 against Hippocampal Apoptosis and Spatial Learning and Memory Deficits in a Rat Model of Vascular Dementia
}

\author{
Shen Yang, ${ }^{1,2}$ Guangan Zhou, ${ }^{2}$ Hong Liu, ${ }^{3}$ Bo Zhang, ${ }^{2}$ Juan Li, \\ Ruiting Cui, ${ }^{2}$ and Yifeng $\mathrm{Du}^{1}$ \\ ${ }^{1}$ Department of Neurology, Shandong Provincial Hospital, Shandong University, Jinan 250012, China \\ ${ }^{2}$ Department of Neurology, Taian Central Hospital, Taian 271000, China \\ ${ }^{3}$ Department of Neurology, Liaocheng Hospital, Liaocheng 252000, China
}

Correspondence should be addressed to Yifeng Du; duyifeng2012@126.com

Received 15 October 2013; Revised 28 November 2013; Accepted 2 December 2013

Academic Editor: Peng Zhang

Copyright (c) 2013 Shen Yang et al. This is an open access article distributed under the Creative Commons Attribution License, which permits unrestricted use, distribution, and reproduction in any medium, provided the original work is properly cited.

\begin{abstract}
Vascular dementia $(\mathrm{VaD})$ is a common age-related neurodegenerative disease resulting from chronic hypoxia. In the present study, we examined the protective effects of p38 MAPK inhibitor SB202190 against hippocampal apoptosis and spatial learning and memory deficits in a chronic hypoperfusion rat model of VaD established by permanent bilateral carotid occlusion (2-VO). Sixty rats were randomly divided into sham-operated, VaD model, and VaD plus SB202190 groups ( $n=20 /$ group). After sham/2-VO surgery, rats were administered 0.1\% DMSO (sham-operated and VaD groups) or SB202190 by intracerebroventricular injection. One week after inhibitor/vehicle treatment, hippocampal p38 MAPK phosphorylation was higher in the model group than in the SB202190 group $(P<0.01)$. Compared to the model group, the SB202190 group exhibited significantly shorter escape latencies in the Morris water maze hidden platform trials $(P<0.01)$ and longer times in the original platform quadrant during probe trials $(P<0.01)$. The SB202190 group also showed significantly reduced neuronal apoptosis in the hippocampus compared to VaD model rats $(P<0.01)$ as well as higher (antiapoptotic) Bcl-2 expression and lower (proapoptotic) caspase- 3 expression $(P<0.01$ for both). In conclusion, blockade of the p38 MAPK signaling pathway by SB202190 following permanent 2-OV reduced apoptosis of hippocampal neurons and rescued spatial learning and memory deficits.
\end{abstract}

\section{Introduction}

Vascular dementia $(\mathrm{VaD})$ is the second most common form of age-related neurological dysfunction in most Western countries after Alzheimer's disease (AD) [1] and may be the most common form in countries with lower rates of $\mathrm{AD}[2,3]$. The core symptom of $\mathrm{VaD}$ is progressive cognitive dysfunction due to cumulative regional brain tissue injury associated with localized cerebrovascular disruptions (microinfarcts or "mini strokes") [4]. The progressive nature of VaD leads to unremitting and largely irreversible deterioration in quality of life and places a heavy emotional and economic burden on families. In countries with aging populations, prevention and treatment of $\mathrm{VaD}$ are a major medical and social priority.
Multiple factors increase the risk of $\mathrm{CD}$, including previous stroke, hypertension, and diabetes [5]. Targeting these risk factors can reduce disease incidence or progression but there are no broadly effective treatments that can reverse the deficits of $\mathrm{VaD}$.

The hippocampal formation, including the hippocampus, dentate gyrus, subiculum, and parahippocampus gyrus, is essential for the formation of declarative memories [6]. Specific regions within this mesial temporal lobe structure are highly susceptible to ischemia-reperfusion injury [7]. Activation of the p38 mitogen-activated protein kinase (p38 MAPK) signaling pathway by hypoxia may initiate neuronal apoptosis, leading to the functional deficits of $\mathrm{VaD}[8]$. In the hippocampus, activation of caspase- 3 and other markers 
of mitochondrial apoptosis in response to hyperosmotic stress was blocked by SB202190, a specific inhibitor of p38 MAPK [9]. Conversely, inhibition of p38 MAPK abolished the cytoprotective effects of ischemic preconditioning [10], suggesting that mild p38 MAPK activation may initiate protective responses while overactivation, which may occur during prolonged hypoxia associated with $\mathrm{VaD}$, may activate cell death pathways. Indeed, activation of MAPK is one of the central signal transduction pathways triggering neuronal apoptosis following neural hypoxia and reperfusion [11-13]. Overexpression of Bcl-2 can effectively inhibit caspase-3 activation and apoptosis [14], and p38 MAPK affects apoptosis by regulating $\mathrm{Bcl}-2$ and caspase- 3 expression [15]. For example, quercetin, a natural MAPK p38 inhibitor, blocked apoptosis in the hippocampus by preventing $\mathrm{Bcl}-2$ downregulation, Bax upregulation, and caspase-3 activation [16]. Moreover, SB202190 can reduce cerebral ischemia-reperfusion injury [17].

No previous study has examined the effect of SB202190 for sustaining hippocampus-dependent spatial memory or the effects of this inhibitor on the expression of apoptotic regulators under chronic ischemia. To these ends, we examined the effects of SB202190 on hippocampal neuron apoptosis, Bcl-2 and caspase- 3 expression, and p38 MAPK phosphoactivation in a rat model of $\mathrm{VaD}$ and tested whether p38 MAPK inhibition can rescue deficits in the hippocampus-dependent Morris water maze test of spatial learning and memory. Our results indicate that blockade of the p38 MAPK signaling pathway can indeed protect hippocampal neurons against chronic ischemic injury and rescue spatial learning and memory deficits, at least in part, by suppressing caspase-3dependent apoptosis.

\section{Materials and Methods}

2.1. Reagents. Rabbit anti-rat Bcl-2, caspase- 3 monoclonal antibodies, SB202190, FITC-conjugated goat anti-rabbit secondary antibody, p-p38 MAPK rabbit anti-rat polyclonal antibody, horseradish peroxidase- (HRP-) conjugated goat anti-rabbit IgG, enhanced chemiluminescence (ECL), bicinchoninic acid (BCA) protein assay, and lysis buffer were purchased from Santa Cruz (CA, USA). $\beta$-Actin was purchased from Yanjing Biological Research Technology Co. (Shanghai, China), an in situ apoptosis detection kit from Roche (USA), and an SABC-FITC immunofluorescence assay kit from Boster (Wuhan, China).

2.2. Experimental Devices. A Morris water maze (DMS-2 type) was obtained from the Chinese Academy of Medical Sciences, a rat stereotaxic apparatus (Jiangwan type I-C) from Huaibei Zhenghua Biologic Apparatus Facilities (Anhui, China), a Radiance 2100 type laser scanning confocal microscope and Gel Doc gel imaging analysis system from Bio-Rad (USA), and a freezing microtome from Leica (CM1900 type, Leica, Germany).

2.3. Animals. Specific pathogen-free (SPF) male Wistar rats ( $n=60$, three month old, $250 \pm 10 \mathrm{~g}$ ) were purchased from the
Experimental Animal Center of Shandong University School of Medicine (China) (Certificate no. SCXK Lu 20090001) and maintained at the Animal House of Taishan Medical University (Shandong, China). The rats were housed at 20$25^{\circ} \mathrm{C}$ and $50 \% \pm 5 \%$ humidity with ad libitum access to food and water under a 12:12 h light/dark cycle for two weeks before experiments. All procedures and animal experiments were approved by the Animal Ethical Committee of Taishan Medical University and conducted in accordance with all state regulations.

2.4. Animal Grouping. The 60 Wistar rats were randomly assigned to the sham-operated group, the VaD model group, and the SB202190 group (20 animals each) using a random number table. The VaD rat model $(n=40)$ was established by separating and ligating the bilateral carotid artery via twovessel occlusion (2-VO). For animals of the sham-operated group $(n=20)$, the bilateral carotid artery was separated using the same methods but without ligation. After recovery, animals of the SB202190 group received intracerebroventricular (ICV) injection of SB202190 (dissolved in 100\% DMSO and then diluted in normal saline (NS) for a final concentration of DMSO of $0.1 \%$ ) and both the VaD model and shamoperated groups received ICV injection of equal volume $0.1 \%$ DMSO. In each group, eight rats were examined in the Morris water maze to assess spatial learning and memory, six rats were sacrificed and brain sections were prepared for TUNEL staining and $\mathrm{Bcl}-2 /$ caspase- 3 immunohistochemistry, and six rats were sacrificed and tissue homogenates were prepared for Western blot assay of phospho-p38 MAPK expression.

2.4.1. Permanent Bilateral Carotid Occlusion (2-VO). Following $12 \mathrm{~h}$ of fasting and $4 \mathrm{~h}$ without water, animals were anesthetized by intraperitoneal (IP) injection of a $10 \%$ chloral hydrate solution at $0.35 \mathrm{~mL} / 100 \mathrm{~g}$ body weight. The injected dose of chloral hydrate was increased when necessary. After loss of the righting reflex, animals were fixed in the supine position on a rat board. An incision was made along the midline of the neck, followed by careful blunt dissection of muscle and connective tissues. The bilateral common carotid artery was isolated and ligated with silk sutures [13]. If normal respiration and heartbeat were maintained, the skin and muscle layers were sutured. Gentamicin $(80,000$ units) was applied to the surgical wound by topical spray and intramuscular injection for three consecutive days. During recovery, the animals were housed and fed in single cages to prevent infection.

2.4.2. Intracerebroventricular Injection. All animals were administered 0.1\% DMSO or SB202190 immediately after sham or 2-VO surgery by ICV injection. Briefly, after anesthesia by IP injection of a $10 \%$ chloral hydrate $(0.35 \mathrm{~mL} / 100 \mathrm{~g}$ body weight), the animal was secured within a Jiangwan I-C rat stereotaxic frame. The site for ICV injection was $0.8 \mathrm{~mm}$ caudal to bregma and $1.5 \mathrm{~mm}$ to the right of the midline. A hole was drilled horizontally with an electric drill and $5 \mu \mathrm{L}$ of $0.1 \%$ DMSO or $10 \mu \mathrm{mol} / \mathrm{L}$ SB202190 solution was injected using a microinjector. The injection was completed 
in $4 \mathrm{~min}$ and the needle kept in position for an additional 2 min. One week after ICV injection, animals were examined in the Morris water maze or sacrificed and brain tissues were prepared for TUNEL staining, immunofluorescence, and Western blot.

2.4.3. Morris Water Maze Test. The Morris water maze setup consisted of a circular pool (diameter: $100 \mathrm{~cm}$, height: $50 \mathrm{~cm}$; wall: blank), a hidden platform, and an overhead video recording system. The pool was arbitrarily divided into northeast, southeast, southwest, and northwest quadrants. Each day, water was poured into the pool to submerge a cylindrical platform at the center of a chosen quadrant $2 \mathrm{~cm}$ below the surface. The water was made cloudy using black polyethylene particles and maintained at $23^{\circ} \mathrm{C} \pm 2^{\circ} \mathrm{C}$. The pool was surrounded by black curtains and maintained in the same position, allowing the rats to locate the platform. A digital camera was placed approximately $1 \mathrm{~m}$ above the center of the pool to record swim paths. The video signals were exported to a computer and analyzed using an automatic image acquisition and analysis system (Drug Research Institute of Chinese Academy of Medical Sciences). Hidden platform trials were conducted for three consecutive days and escape latencies recorded as an index of spatial learning. Memory for platform location was tested on the fourth day by a probe test in which the platform was removed and time spent in the original platform quadrant was recorded [18].

2.4.4. TUNEL Staining. In each group, six rats were decapitated and hippocampal tissue was isolated and immediately frozen in liquid nitrogen. Frozen sections were prepared at approximately $10 \mu \mathrm{m}$ and fixed in freshly prepared $4 \%$ paraformaldehyde at room temperature for $30 \mathrm{~min}$. After washing with PBS, a blocking agent $\left(0.3 \% \mathrm{H}_{2} \mathrm{O}_{2}\right.$ methanol solution) was added dropwise and slices were incubated at room temperature for $30 \mathrm{~min}$. Slices were then washed in PBS again and permeabilized by drop-wise addition of icecold $0.1 \%$ Triton X-100 in $0.1 \%$ citric acid solution for $2 \mathrm{~min}$. Slices were washed with PBS twice, excess liquid was removed using tissue paper, and $50 \mu \mathrm{L}$ of TUNEL reaction mixture was added drop-wise, followed by incubation in a wet box at $37^{\circ} \mathrm{C}$ for $60 \mathrm{~min}$. Finally, the slices were washed three times with PBS, mounted with a $1: 1$ glycerol PBS solution, and examined under a confocal laser scanning microscope. Images were taken using the Lasersharp 2000 software package. For each animal, slices were randomly examined and the average number of TUNEL-positive cells was determined from 30 random fields of view [19].

2.4.5. Immunofluorescence. Hippocampal tissue samples were isolated and immediately frozen in liquid nitrogen. Frozen sections were prepared at approximately $8 \mu \mathrm{m}$ on a cryostat microtome and then fixed in freshly prepared $4 \%$ paraformaldehyde at room temperature for $30 \mathrm{~min}$. After antigen retrieval, $0.4 \%$ Triton X-100 and $1 \%$ bovine serum albumin were added drop-wise, followed by incubation at room temperature for $1 \mathrm{~h}$. Goat serum blocking solution was added drop-wise and slices were incubated for $5 \mathrm{~h}$.
After removing excess blocking solution, a solution of 1:200 rabbit anti-rat $\mathrm{Bcl}-2$ or caspase-3 monoclonal antibody in $0.01 \mathrm{~mol} / \mathrm{L}$ PBS was added drop-wise and slices were incubated at $4^{\circ} \mathrm{C}$ overnight. Thereafter, the primary antibody was washed off with PBS (three times), and the FITC-conjugated secondary antibody was added. The immunolabeled slices were mounted with 1:1 diluted glycerol in PBS and FITC-positive cells were detected by confocal laser scanning microscopy. Both fluorescence intensity and the number of FITC-labeled cells were determined in 30 random fields of view [20].

2.4.6. Western Blotting. Hippocampal tissue was isolated and homogenized in lysis buffer (in mM: 50 Tris- $\mathrm{Cl} \mathrm{pH} \mathrm{7.5,} 150$ $\mathrm{NaCl}, 40 \mathrm{KF}, 5$ EDTA, 5 EGTA, 1 orthovanadate, and 1 PMSF with $0.1 \%$ SDS, $0.1 \%$ aprotinin, $0.1 \%$ sodium deoxycholate, and $0.5 \% \mathrm{NP}-40)$ on ice. The homogenate was centrifuged $\left(13,000 \times \mathrm{g}, 15 \mathrm{~min}, 4^{\circ} \mathrm{C}\right)$ and the total supernatant protein was measured by BCA assay. Then, $50 \mu \mathrm{g}$ of protein per gel lane was separated by $10 \%$ SDS-PAGE and proteins were transferred to nitrocellulose membranes (Santa Cruz). Membranes were blocked by a one-hour incubation in TBS containing $10 \%$ skim milk powder and $0.05 \%$ Tween 20 (TBST). The blocked membranes were incubated in primary antibody (1:1000 p-p38 MAPK rabbit anti-rat polyclonal antibody or $1: 2000 \beta$-actin as an internal reference) at $4^{\circ} \mathrm{C}$ for $2 \mathrm{~h}$ and then with the secondary antibody (HRP-conjugated goat anti-rabbit IgG) at room temperature for $1 \mathrm{~h}$. Immunolabeling was then visualized by ECL. The relative intensity of each protein band was measured by the Gel Doc gel imaging analysis system. Results are presented as the optical density ratio of p-p38 MAPK and $\beta$-actin.

2.4.7. Statistical Analyses. All statistical tests were conducted using SPSS15.0 software (SPSS Inc., Chicago, IL, USA). Data are expressed as means \pm standard deviation (SD). Statistical significance was evaluated by one-way analysis of variance (ANOVA) with Tukey's test for post hoc analysis. A $P<0.05$ was considered statistically significant.

\section{Results}

3.1. Effect of Chronic Ischemia on p38 MAPK Phosphoactivation in the Hippocampus of VaD Rat Model. Rats were subjected to either permanent bilateral carotid artery occlusion (VaD model group, $n=20$; SB202190 p38 MAPK inhibitor group, $n=20)$ or sham operation $(n=20)$ and then immediately administered 0.1\% DMSO (sham-operated and VaD model groups) or SB202190 by ICV injection. One week after ICV injection, we observed significant differences in hippocampal p-p38 MAPK expression levels among the treatment groups $(P<0.01)$, with significantly higher expression in the $\mathrm{VaD}$ model group compared to the shamoperated group $(0.974 \pm 0.110$ versus $0.254 \pm 0.021 ; P<0.01)$. Compared to the model group, p-p38 MAPK expression was significantly reduced by SB202190 $(0.432 \pm 0.039)(P<0.01)$ (Figure 1). 

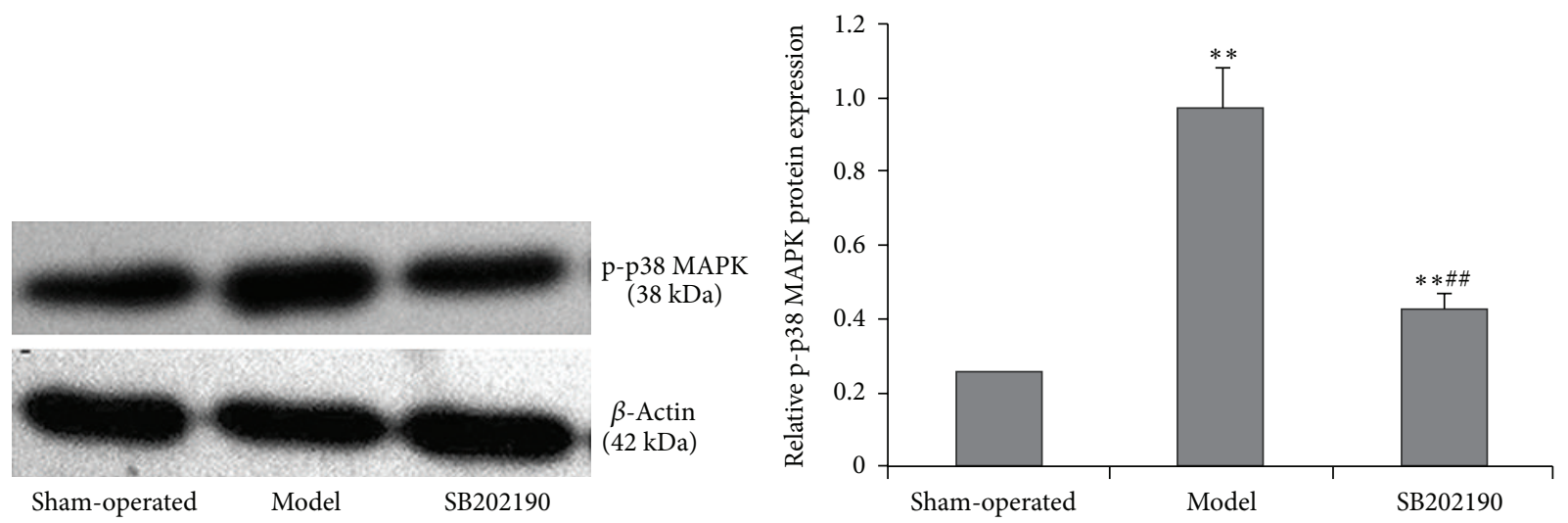

FIGURE 1: Effect of the p38 MAPK inhibitor SB202190 on expression of p-p38 MAPK in the hippocampus of a vascular dementia (VaD) rat model. Expression of p-p38 MAPK was determined by Western blot and protein expression normalized to $\beta$-actin. The data are expressed as means \pm standard deviation (SD) $(n=6) ;{ }^{* *} P<0.01$ versus sham-operated group, ${ }^{\# \#} P<0.01$ SB202190 group versus model group.

TABLE 1: Comparison of escape latencies in Morris water maze learning trials among treatment groups.

\begin{tabular}{lccr}
\hline Groups & Day 1 & Day 2 & Day 3 \\
\hline Sham-operated & $24.17 \pm 4.12$ & $21.59 \pm 5.13$ & $16.83 \pm 3.77^{\Delta \Delta}$ \\
Model & $82.71 \pm 8.27^{* *}$ & $80.36 \pm 9.65^{* *}$ & $77.74 \pm 6.33^{* *}$ \\
SB202190 & $48.72 \pm 7.01^{* * \# \#}$ & $42.41 \pm 4.06^{* * \# \#}$ & $40.34 \pm 2.46^{* * \# \#}$ \\
\hline
\end{tabular}

Note. Data are expressed as means \pm standard deviation (SD) $(n=8) ;{ }^{* *} P<0.01$ versus sham-operated group, ${ }^{\# \#} P<0.01$ SB202190 group versus model group, ${ }^{\Delta} P<0.05,{ }^{\Delta \Delta} P<0.01$ versus Day 1 .

\subsection{Protective Effects of SB202190 against Spatial Learning} and Memory Deficits in a Rat Model of VaD. One week after ICV injection, hippocampus-dependent spatial learning was examined by the MWM hidden platform test. On each of the three testing days, escape latency was significantly longer in the model group than that in the sham-operated group (all $P<0.01$ ), indicative of hippocampus-dependent spatial learning deficits. Latencies were significantly shorter in rats injected with SB202190 compared to the VaD model group on each day of testing (all $P<0.01$ ). Over the 3 days of training, mean escape latency in the sham-operated rats decreased from $24.17 \pm 4.12 \mathrm{~s}$ to $16.83 \pm 3.77 \mathrm{~s}(P<0.01)$, indicating spatial learning, whilst latency in the VaD model group was not significantly reduced by training (from $82.71 \pm 8.27 \mathrm{~s}$ to $77.74 \pm 6.33 \mathrm{~s}, P>0.05)$. Escape latencies in the SB202190 group decreased from $48.72 \pm 7.01 \mathrm{~s}$ to $40.34 \pm 2.46 \mathrm{~s}(P<$ 0.05 ), indicating spatial learning improvement (Table 1).

On day 4 , the hidden platform was removed from the MWM in order to conduct a probe test of spatial memory. The time spent in the original platform quadrant was significantly longer in the $\mathrm{VaD}$ model group than in the sham-operated group $(P<0.01)$, while target quadrant time was lower in the SB202190 group than in the VaD model group $(P<$ 0.01 ), indicating partial rescue to spatial memory (Table 2$)$. In contrast, there was no difference in swim speed among groups, indicating that motor impairments did not account for these differences in training or probe test performance. Thus, prolonged latencies in the training trial are indicative of disrupted spatial learning in $\mathrm{VaD}$ model rats, while reduced time in the target quadrant is indicative of impaired hippocampus-dependent spatial memory. Moreover, reduced escape latency and longer target quadrant time in the SB202190 group compared to the model group indicate that blockade of p38 MAPK signaling partially preserves spatial learning and memory during chronic ischemia.

3.3. SB202190 Reduced Apoptosis of Hippocampal Neurons in a Rat Model of VaD. One week after ICV injection, TUNELpositive (green fluorescent) cell numbers were significantly increased in model and SB202190 groups compared to the sham-operated group (both $P<0.01$ ), but the number of TUNEL-positive cells was significantly lower in the SB202190 group $(P<0.01)$ (Figure 2$)$. Thus, inhibition of p38 MAPK protected hippocampal neurons against chronic ischemiainduced apoptosis.

\subsection{Effects of SB202190 on Bcl-2 and Caspase-3 Expressions} in the Hippocampus of VaD Rat Model. One week after ICV injection, only faint Bcl-2 immunofluorescence was observed in hippocampal slices prepared from sham-operated rats; by comparison, the Bcl-2 immunofluorescence intensity was significantly higher in slices from VaD model and SB202190 groups but was significantly higher in the SB202190 group (all $P<0.01$ ) (Figure 3(a)). These differences in immunofluorescence intensity were paralleled by changes in Bcl-2-positive cell number, which was significantly higher in hippocampal slices from SB202190 group rats from than VaD model group rats $(P<0.01)$ (Figure $3(\mathrm{~b})$ ), suggesting that inhibition of $\mathrm{p} 38$ MAPK may reduce apoptosis by enhancing expression of this antiapoptotic protein. 
TABLE 2: Comparison of target quadrant time and swim speed during memory probe trials among treatment groups.

\begin{tabular}{lcc}
\hline Groups & Time spent in the original platform quadrant $(\mathrm{s})$ & Speed $(\mathrm{cm} / \mathrm{s})$ \\
\hline Sham-operated & $35.21 \pm 3.78$ & 13.46 \\
Model & $18.67 \pm 5.39^{* *}$ & 12.19 \\
SB202190 & $26.45 \pm 4.66^{* * \# \#}$ & 12.28 \\
\hline
\end{tabular}

Note. Data expressed as means $\pm \mathrm{SD}(n=8) ;{ }^{* *} P<0.01$ versus sham-operated group, ${ }^{\# \#} P<0.01$ SB202190 group versus model group.

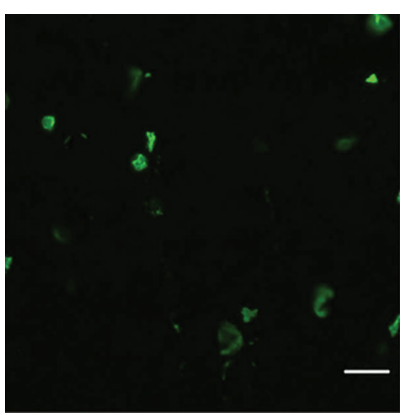

Sham-operated

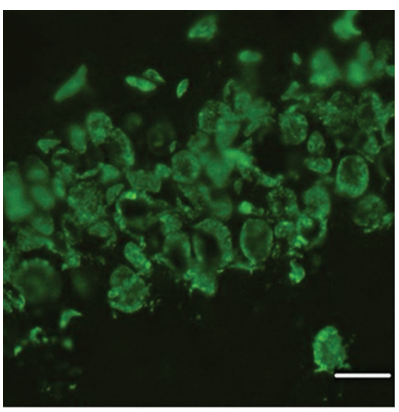

Model

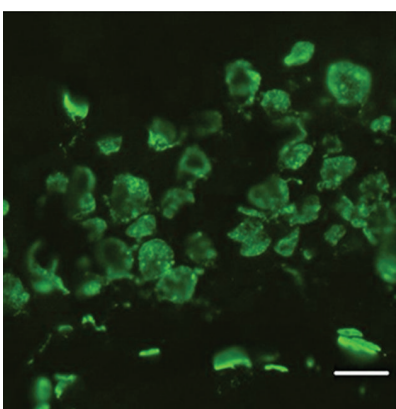

SB202190

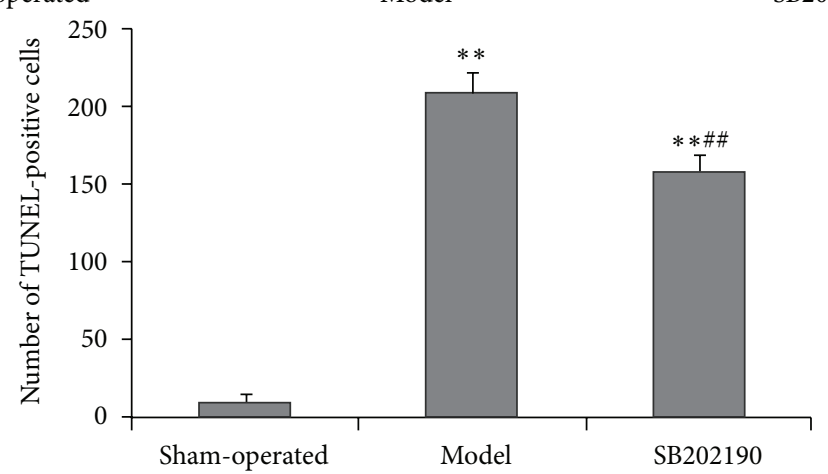

FIGURE 2: Effect of SB202190 on neural apoptosis in the hippocampus of the VaD rat model. Neural apoptosis was assessed by TUNEL (magnification $\times 40)$. Data are expressed as means $\pm \mathrm{SD}(n=6) ;{ }^{* *} P<0.01$ versus sham-operated group, ${ }^{\# \#} P<0.01$ SB202190 group versus model group.

One week after ICV injection, only faint caspase-3 immunofluorescence was detected in hippocampal slices from sham-operated rats compared to $\mathrm{VaD}$ model group rats, indicating that chronic ischemia may activate the mitochondria-dependent apoptosis pathway. However, caspase-3 immunofluorescence was significantly lower in slices from the SB202190 group compared to the model group $(P<0.01)$ (Figure $4(\mathrm{a})$ ), and again this increase in immunofluorescence was paralleled by an increase in caspase-3-positive cell number $(P<0.01)$ (Figure 4(b)).

\section{Discussion}

Vascular dementia is a major cause of senile dementia, accounting for $30 \%-50 \%$ of cases by region [3]. Moreover, $\mathrm{VaD}$ is often comorbid with $\mathrm{AD}$, and these two forms of dementia may act synergistically to damage neural tissue and impair cognition [1]. The hippocampus is highly sensitive to ischemia and hypoxia; indeed, it usually sustains the greatest injury as evidenced by infarct volume during chronic cerebral hypoperfusion [21]. Chronic cerebral hypoperfusion is the primary cause of $\mathrm{VaD}$. The present study established a chronic cerebral hypoperfusion model by bilateral carotid artery ligation and demonstrated severe disruption of hippocampusdependent spatial learning and memory, strong phosphoactivation of p38 MAPK, and enhanced activation of caspase-3dependent apoptosis. Most importantly, these neurocellular and cognitive defects were partially reversed by blockade of p38 MAPK. Thus, activation of p38 MAPK by chronic ischemia activates apoptosis in the hippocampus, which ultimately disrupts spatial learning. Duarte et al. [22] also found p38 MAPK activation during ischemia-reperfusion, suggesting that p38 MAPK is also a potential therapeutic target for stroke. Thus, neuroprotection by p38 MAPK inhibition is a possible therapeutic strategy for preserving neuronal viability and cognition in patients with cerebrovascular disorders.

A myriad of animal models of neurovascular disease and acute stroke have been established to elucidate pathogenic mechanisms and identify possible neuroprotective strategies. Such models have helped reveal glutamate-mediated excitotoxicity, calcium dysregulation, oxidative stress, acidosis, edema, mitochondria-dependent apoptosis, proteolysis, and inflammation as major mutually reenforcing pathogenic processes leading to neural damage during ischemia-reperfusion 


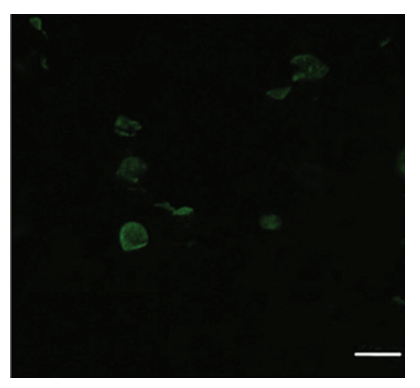

Sham-operated

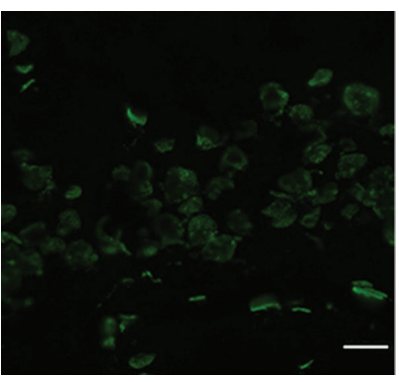

Model

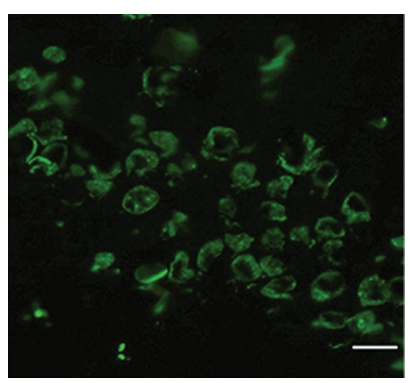

SB202190

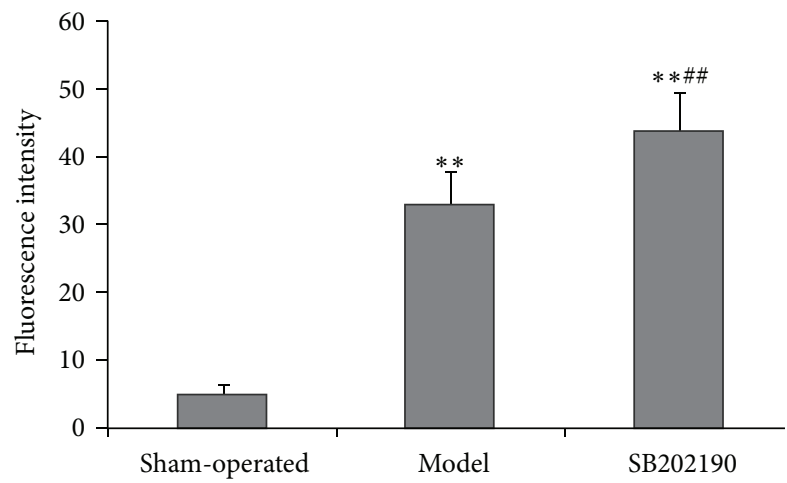

(a)

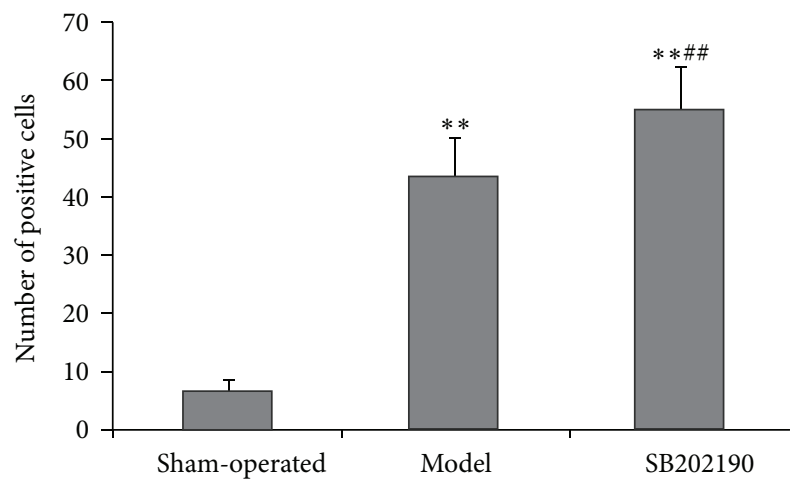

(b)

FIGURE 3: Effect of SB202190 on expression of Bcl-2 in the hippocampus of the VaD rat model. Expression of Bcl-2 was determined by immunofluorescence staining (mMagnification $\times 40$ ). Green: FITC. (a) Fluorescence intensity; (b) number of positive cells. The data are expressed as means \pm SD $(n=6) ;{ }^{* *} P<0.01$ versus sham-operated group, ${ }^{\# \#} P<0.01$ SB202190 group versus model group.

[23]. Conversely, few compounds identified as neuroprotectants in animals have proven effective in clinical practice [24], so these models either do not replicate human stroke pathogenesis, are more resistant to ischemia, or both. The first chronic cerebral hypoperfusion model used stents of different sizes to partially occlude cerebral blood flow in mice [25] and resulted in delayed infarct development in both white and gray matter. In the current study, we used total occlusion as the rat brain is generally less sensitive to ischemia compared to humans, at least in most experimental conditions $[23,26,27]$. First, rats used in stroke modeling are almost always young, healthy, genetically homogenous SPF males, while human stroke patients are usually elderly and have multiple comorbidities and risk factors [27]. Second, brain size, length and structure of perforating arteries, and gray to white matter ratio (lower in humans) influence both stroke resistance and the penetration of potentially neuroprotective drugs [26]. Third, the temporal window for reperfusion or neuroprotectant treatment may not be achieved in humans [23], while administration of possible neuroprotectants in studies such as ours is predetermined. Other possible reasons for differences in human ischemic tolerance included difference in astroglia [28], which can be either neuroprotective or facilitators of neuronal death and impeders of recovery under specific conditions [29]. Finally, hypoperfusion results in mild hypothermia, which is also broadly neuroprotective [23]. Considering that even strains of rats show marked differences in ischemic vulnerability
[30], there are obviously a multitude of additional factors contributing to this differential stroke sensitivity between rats and humans. In light of these considerations, potential therapies should ultimately be tested in both young and older rats as well as in rats genetically prone to spontaneous stroke. Furthermore, multiple stroke induction mechanisms should be tested. Now that we and others [31] have identified p38 MAPK inhibitors as potential therapeutics, such extensive studies may be warranted.

Much is known of the mechanism leading from p38 MAPK activation to neurological disease. p38 MAPK is a member of the larger MAPK family involved in pathophysiological processes like inflammation, stress responses, and hyperproliferation $[32,33]$. Once activated by upstream phosphorylation, p38 MAPK further activates downstream transcription factors, MAPK-activated protein kinases, and caspases. SB202190 is a pyridinyl imidazole derivative that can effectively and selectively inhibit p38 MAPK activation by preventing its binding to ATP. Fernandez et al. [34] demonstrated that SB202190 can alleviate learning and memory impairment in rats with chronic cerebral hypoperfusionischemia, a state resembling $\mathrm{VaD}$. The present study was designed to confirm these results and elucidate the underlying molecular mechanisms for this effect.

The escape latency to the hidden platform in the MWM was significantly shorter and the time spent in the platform (target) quadrant is longer in the SB202190-pretreated VaD group compared to the vehicle-treated $\mathrm{VaD}$ group. Zhao et al. 


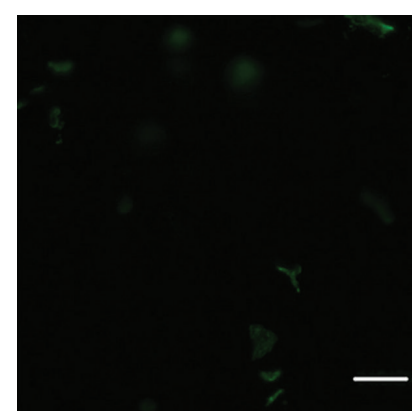

Sham-operated

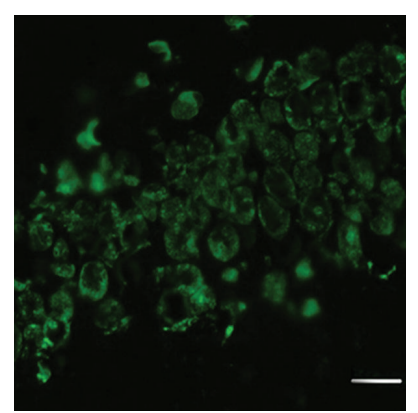

Model

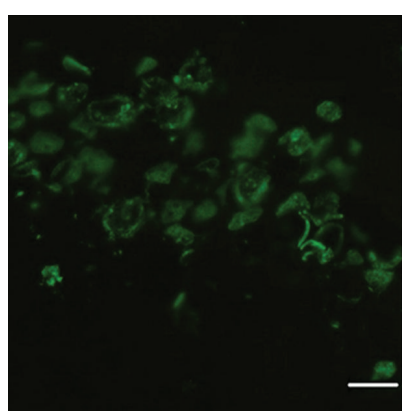

SB202190

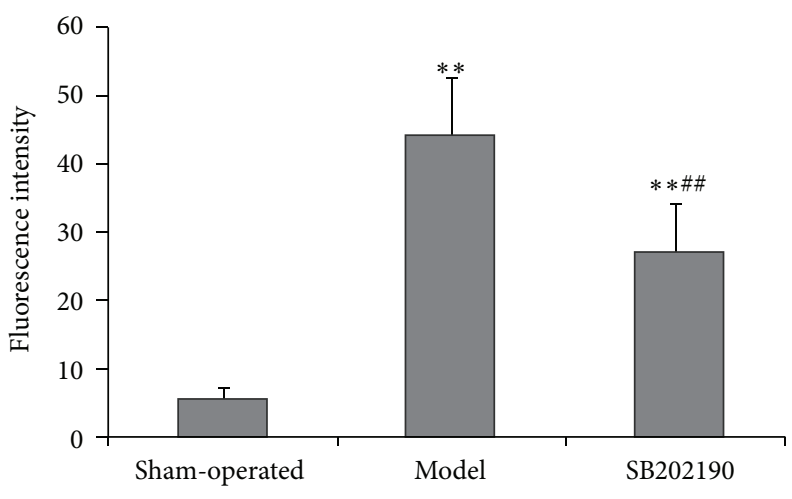

(a)

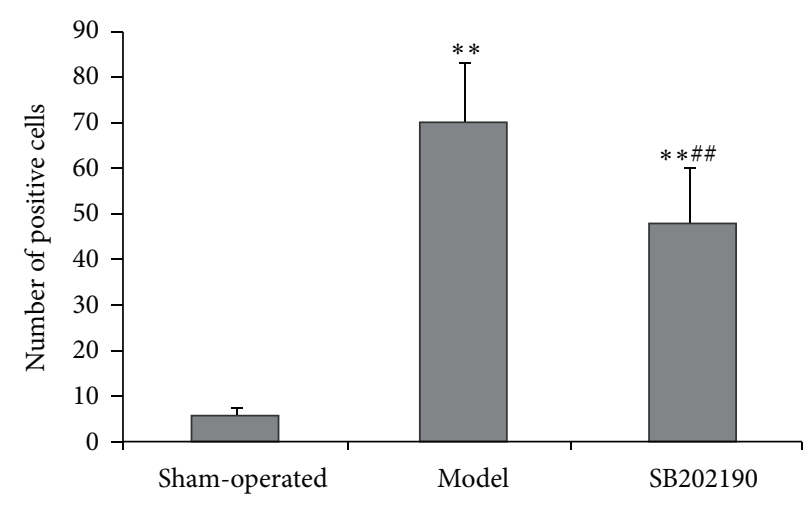

(b)

FIGURE 4: Effect of SB202190 on expression of caspase- 3 in the hippocampus of the VaD rat model. Expression of caspase- 3 was determined by immunofluorescence staining. (magnification $\times 40$ ). Green: FITC. (a) Fluorescence intensity; (b) number of positive cells. Data are expressed as means $\pm \mathrm{SD}(n=6) ;{ }^{* *} P<0.01$ versus sham-operated group, ${ }^{\# \#} P<0.01$ SB202190 group versus model group.

[35] reported that $\mathrm{p} 38$ MAPK inhibition improved learning and memory in rats subjected to ischemia-reperfusion by regulating the expression of pro- and antiapoptotic factors. Similarly, expression of antiapoptotic $\mathrm{Bcl}-2$ was enhanced in SB202190-treated VaD rats while caspase-3 expression was reduced compared to vehicle-treated $\mathrm{VaD}$ rats. Apoptosis is strictly controlled by multiple gene families, including the Bcl-2 and caspase families, as well as by c-myc and p53. The reciprocal regulation of apoptosis by $\mathrm{Bcl}-2$ and caspase- 3 is well described [36]. Increasing Bcl-2 expression in brain can reduce cerebral infarct volume by protecting neurons surrounding the ischemic focus (penumbra) [37]. Yang et al. found that ICV injection of a caspase-3 inhibitor reduced infarct area and neural apoptosis [38]. Bcl-2 is an upstream inhibitor of capsase-3, the primary effector of apoptosis. After stress-induced phosphoactivation, p38 MAPK activates gene expression patterns that can alternately induce proliferation, differentiation, cytokine synthesis, or caspase-3-dependent apoptosis. Chen et al. [17], and Liu et al. [39] showed that the learning and memory capacities of $\mathrm{VaD}$ rats were closely related to p38 MAPK expression. Results from the present study confirmed these findings and further demonstrate that the p38 MAPK pathway regulates expression of $\mathrm{Bcl}-2$ and caspase-3. SB202190 inhibits neuronal apoptosis at least in part by sustaining overexpression of $\mathrm{Bcl}-2$, thereby inhibiting pathological processes leading to caspase- 3 activation, such as activation of the mitochondrial permeability transition pore
(mPTP), loss of inner membrane potential, and cytochrome $c$ release.

In $\mathrm{VaD}$, the protective effects of SB202190 against hippocampal neuronal apoptosis and spatial learning and memory deficits were likely due in part to suppression of the caspase- 3 apoptotic pathway by Bcl-2 upregulation. While many of these protective effects were moderate, it is important to reemphasize that multiple interacting pathogenic processes contribute to stroke-induced neurodegeneration. Thus, inhibition of p38 MAKP may benefit stroke only when used in combination with other agents, such as thrombolytics, antioxidants, calcium chelators, and anti-inflammatories [24]. It is also clear that the p38 MAPK signaling pathway has both beneficial and deleterious effects on the nervous system during stress. Identifying those conditions in which p38 MAPK contributes to neuronal damage may allow for the judicious use of nontoxic inhibitors for treatment of neurological diseases, such as $\mathrm{VaD}$ and acute stroke.

\section{Conflict of Interests}

The authors declare that they have no conflict of interests.

\section{Authors' Contribution}

All authors have read and approved the final paper. 


\section{References}

[1] L. Battistin and A. Cagnin, "Vascular cognitive disorder. A biological and clinical overview," Neurochemical Research, vol. 35, no. 12, pp. 1933-1938, 2010.

[2] J. A. Catindig, N. Venketasubramanian, M. K. Ikram, and C. Chen, "Epidemiology of dementia in Asia: insights on prevalence, trends and novel risk factors," Journal of the Neurological Sciences, vol. 321, no. 1-2, pp. 11-16, 2012.

[3] N. Venketasubramanian, S. Sahadevan, E. H. Kua, C. P. L. Chen, and T.-P. Ng, "Interethnic differences in dementia epidemiology: global and Asia-Pacific perspectives," Dementia and Geriatric Cognitive Disorders, vol. 30, no. 6, pp. 492-498, 2011.

[4] M. Brundel, J. De Bresser, J. J. Van Dillen, L. J. Kappelle, and G. J. Biessels, "Cerebral microinfarcts: a systematic review of neuropathological studies," Journal of Cerebral Blood Flow and Metabolism, vol. 32, no. 3, pp. 425-436, 2012.

[5] F. Rincon and C. B. Wright, "Vascular cognitive impairment," Current Opinion in Neurology, vol. 26, no. 1, pp. 29-36, 2013.

[6] G. Buzsaki and E. I. Moser, "Memory, navigation and theta rhythm in the hippocampal-entorhinal system," Nature Neuroscience, vol. 16, no. 2, pp. 130-138, 2013.

[7] M. A. Philbert, M. L. Billingsley, and K. R. Reuhl, "Mechanisms of injury in the central nervous system," Toxicologic Pathology, vol. 28 , no. 1, pp. 43-53, 2000.

[8] M. Fang, J. Li, S. C. Tiu, L. Zhang, M. Wang, and D. T. Yew, "N-methyl-D-aspartate receptor and apoptosis in Alzheimer's disease and multiinfarct dementia," Journal of Neuroscience Research, vol. 81, no. 2, pp. 269-274, 2005.

[9] J. M. Niswander and L. A. Dokas, "Hyperosmotic stressinduced caspase- 3 activation is mediated by $\mathrm{p} 38$ MAPK in the hippocampus," Brain Research, vol. 1186, no. 1, pp. 1-11, 2007.

[10] X. D. Wu, Z. Y. Zhang, S. Sun et al., "Hypoxic preconditioning protects microvascular endothelial cells against hypoxia/reoxygenation injury by attenuating endoplasmic reticulum stress," Apoptosis, vol. 18, no. 1, pp. 85-98, 2013.

[11] E. A. Irving, F. C. Barone, A. D. Reith, S. J. Hadingham, and A. A. Parsons, "Differential activation of MAPK/ERK and p38/SAPK in neurones and glia following focal cerebral ischaemia in the rat," Molecular Brain Research, vol. 77, no. 1, pp. 65-75, 2000.

[12] J. J. Legos, J. A. Erhardt, R. F. White et al., "SB 239063, a novel p38 inhibitor, attenuates early neuronal injury following ischemia," Brain Research, vol. 892, no. 1, pp. 70-77, 2001.

[13] X. L. Wang, P. Y. Lv, and Z. C. Guo, "The study of expression of p38 MAPK mRNA in neuron of hippocampus in mice with vascular dementia," Journal of Apoplexy and Nervous Diseases, vol. 2, p. 014, 2008.

[14] T. Sasaki, K. Kitagawa, Y. Yagita et al., "Bcl2 enhances survival of newborn neurons in the normal and ischemic hippocampus," Journal of Neuroscience Research, vol. 84, no. 6, pp. 1187-1196, 2006.

[15] G. Ashabi, S. Z. Alamdary, M. Ramin, and F. Khodagholi, "Reduction of hippocampal apoptosis by intracerebroventricular administration of extracellular signal-regulated protein kinase and/or p38 inhibitors in amyloid beta rat model of Alzheimer's disease: involvement of nuclear-related factor2 and nuclear factor- $\kappa \mathrm{B}$, , Basic \& Clinical Pharmacology \& Toxicology, vol. 112, no. 3, pp. 145-155, 2013.
[16] A. Sunkaria, D. Verma, and K. Gill, "Quercetin attenuates aluminum-induced apoptosis in rat hippocampus, by preventing cytochrome $\mathrm{c}$ translocation, Bcl-2 decrease, bax elevation, caspase- 3 and p53 activation," Free Radical Biology and Medicine, vol. 53, pp. S44-S45, 2012.

[17] M. Chen, H.-Y. Sun, S.-J. Li, M. Das, J.-M. Kong, and T.M. Gao, "Nitric oxide as an upstream signal of p38 mediates hypoxia/reoxygenation-induced neuronal death," NeuroSignals, vol. 17, no. 2, pp. 162-168, 2009.

[18] R. D’Hooge and P. P. De Deyn, “Applications of the Morris water maze in the study of learning and memory," Brain Research Reviews, vol. 36, no. 1, pp. 60-90, 2001.

[19] K. Kyrylkova, S. Kyryachenko, M. Leid, and C. Kioussi, "Detection of apoptosis by TUNEL assay," Methods Molecular Biology, vol. 887, pp. 41-47, 2012.

[20] M. Barbierato, C. Argentini, and S. D. Skaper, "Indirect immunofluorescence staining of cultured neural cells," Methods in Molecular Biology, vol. 846, pp. 235-246, 2012.

[21] L. H. S. Sekhon, M. K. Morgan, I. Spence, and N. C. Weber, "Chronic cerebral hypoperfusion: pathological and behavioral consequences," Neurosurgery, vol. 40, no. 3, pp. 548-556, 1997.

[22] S. Duarte, X. D. Shen, C. Fondevila, R. W. Busuttil, and A. J. Coito, "Fibronectin-alpha4betal interactions in hepatic cold ischemia and reperfusion injury: regulation of MMP-9 and MT1-MMP via the p38 MAPK pathway," American Journal of Transplantation, vol. 12, no. 10, pp. 2689-2699, 2012.

[23] T. M. Woodruff, J. Thundyil, S.-C. Tang, C. G. Sobey, S. M. Taylor, and T. V. Arumugam, "Pathophysiology, treatment, and animal and cellular models of human ischemic stroke," Molecular Neurodegeneration, vol. 6, no. 1, article 11, 2011.

[24] S. Y. Xu and S. Y. Pan, "The failure of animal models of neuroprotection in acute ischemic stroke to translate to clinical efficacy," Medical Science Monitor Basic Research, vol. 19, pp. 3745, 2013.

[25] M. Shibata, R. Ohtani, M. Ihara, and H. Tomimoto, "White matter lesions and glial activation in a novel mouse model of chronic cerebral hypoperfusion," Stroke, vol. 35, no. 11, pp. 25982603, 2004.

[26] P. R. Krafft, E. L. Bailey, T. Lekic et al., "Etiology of stroke and choice of models," International Journal of Stroke, vol. 7, no. 5, pp. 398-406, 2012.

[27] J. B. Casals, N. C. G. Pieri, M. L. T. Feitosa et al., "The use of animal models for stroke research: a review," Comparative Medicine, vol. 61, no. 4, pp. 305-313, 2011.

[28] N. A. Oberheim, T. Takano, X. Han et al., "Uniquely hominid features of adult human astrocytes," Journal of Neuroscience, vol. 29, no. 10, pp. 3276-3287, 2009.

[29] G. Barreto, R. E. White, Y. Ouyang, L. Xu, and R. G. Giffard, "Astrocytes: targets for neuroprotection in stroke," Central Nervous System Agents in Medicinal Chemistry, vol. 11, no. 2, pp. 164-173, 2011.

[30] M. Walberer, E. Stolz, C. Müller et al., "Experimental stroke: ischaemic lesion volume and oedema formation differ among rat strains (a comparison between Wistar and Sprague-Dawley rats using MRI)," Laboratory Animals, vol. 40, no. 1, pp. 1-8, 2006.

[31] F. C. Barone, E. A. Irving, A. M. Ray et al., "Inhibition of p38 mitogen-activated protein kinase provides neuroprotection in cerebral focal ischemia," Medicinal Research Reviews, vol. 21, no. 2, pp. 129-145, 2001. 
[32] K. Takeda and H. Ichiijo, "Neuronal p38 MAPK signalling: an emerging regulator of cell fate and function in the nervous system," Genes to Cells, vol. 7, no. 11, pp. 1099-1111, 2002.

[33] S. Yasuda, H. Sugiura, H. Tanaka, S. Takigami, and K. Yamagata, "p38 MAP kinase inhibitors as potential therapeutic drugs for neural diseases," Central Nervous System Agents in Medicinal Chemistry, vol. 11, no. 1, pp. 45-59, 2011.

[34] F. Fernandez, I. Soon, Z. Li et al., "Wip1 phosphatase positively modulates dendritic spine morphology and memory processes through the p38MAPK signaling pathway," Cell Migration Assays, vol. 6, no. 4, pp. 333-343, 2012.

[35] Y. N. Zhao, J. M. Li, C. W. An, X. Y. Zhang, S. X. $\mathrm{Li}$, and C. X. Cheng, "Effect of Buyanghuanwu recipe on p38MAPK/neuronal apoptosis signal pathway and learningmemory function after forebrain ischemia-reperfusion injury in gerbils," Pharmacology and Clinics of Chinese Materia Medica, vol. 2, p. 003, 2010.

[36] F. Hua, M. G. Cornejo, M. H. Cardone, C. L. Stokes, and D. A. Lauffenburger, "Effects of Bcl-2 levels on Fas signaling-induced caspase-3 activation: molecular genetic tests of computational model predictions," The Journal of Immunology, vol. 175, no. 2, pp. 985-995, 2005.

[37] R. Hata, F. Gillardon, T. M. Michaelidis, and K.-A. Hossmann, "Targeted disruption of the bcl-2 gene in mice exacerbates focal ischemic brain injury," Metabolic Brain Disease, vol. 14, no. 2, pp. $117-124,1999$.

[38] Y. Yang, S.-M. Zhang, S.-Y. Fan et al., "Effects of activation of caspase-3 in cerebral ischemic reperfusion injury," Zhonghua yi xue za zhi, vol. 84, no. 12, pp. 1035-1037, 2004.

[39] B. Liu, W. J. Mao, A. C. Li et al., "Effect of shenxiong huayu capsule on expression of p38MAPK in CA1 area of hippocampus in vascular dementia rats," Chinese Journal of Experimental Traditional Medical Formulae, vol. 8, pp. 161-165, 2010. 


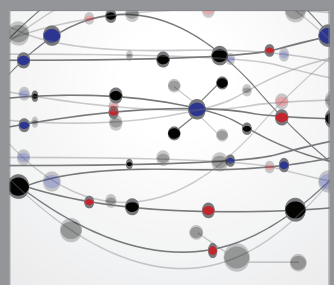

The Scientific World Journal
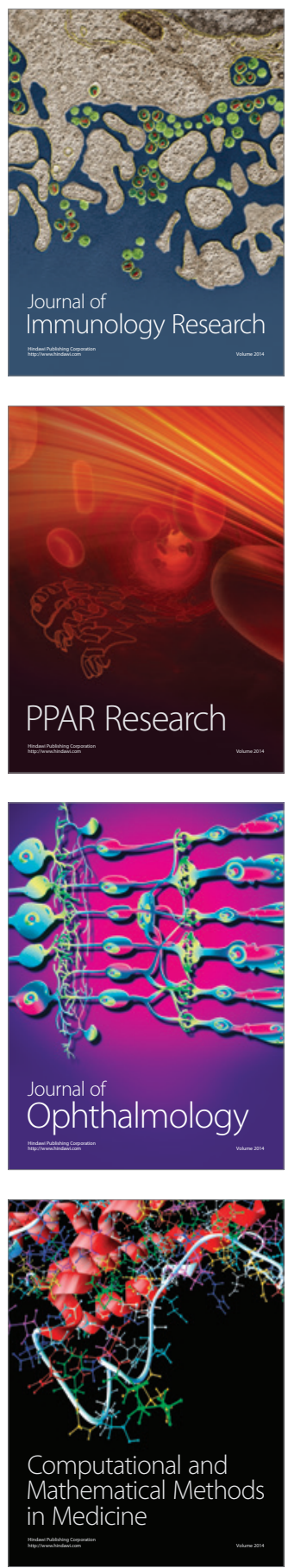

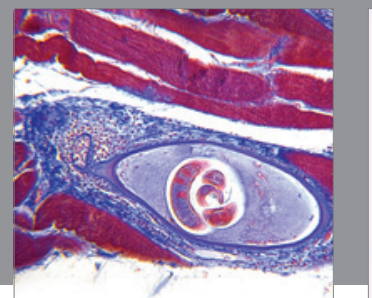

Gastroenterology

Research and Practice
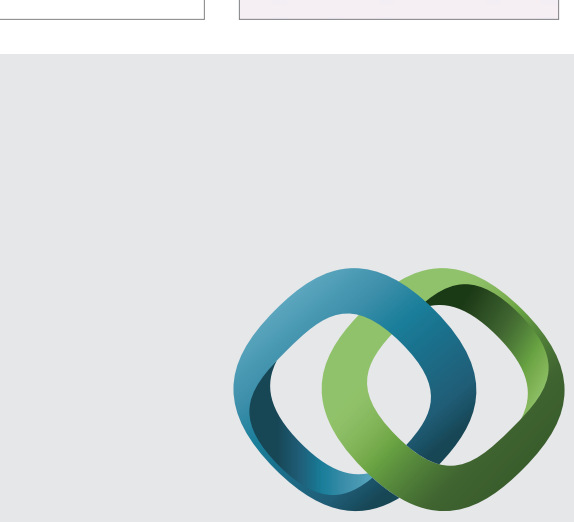

\section{Hindawi}

Submit your manuscripts at

http://www.hindawi.com
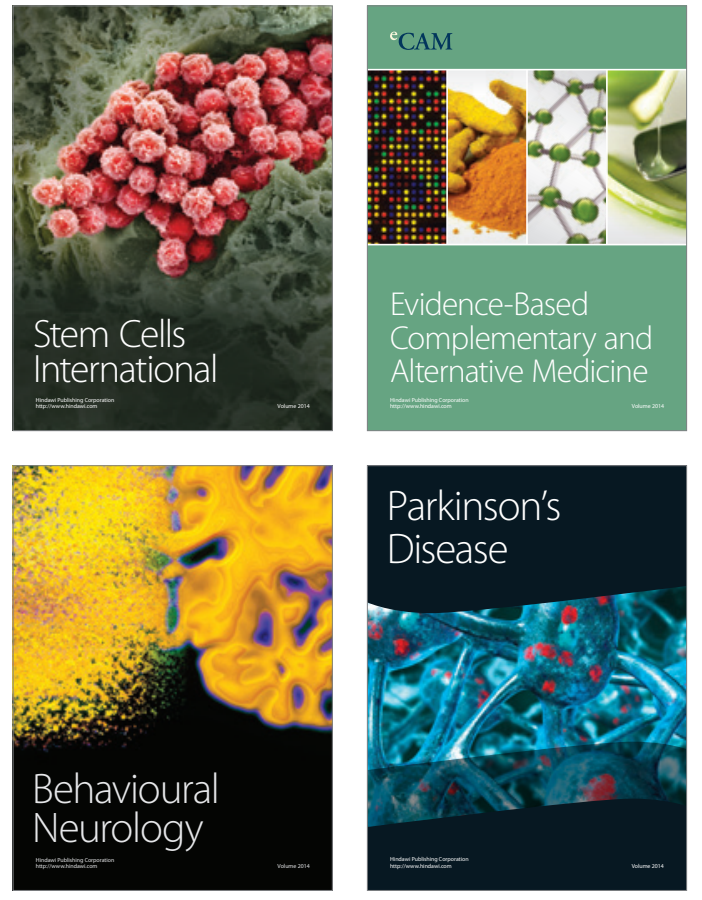
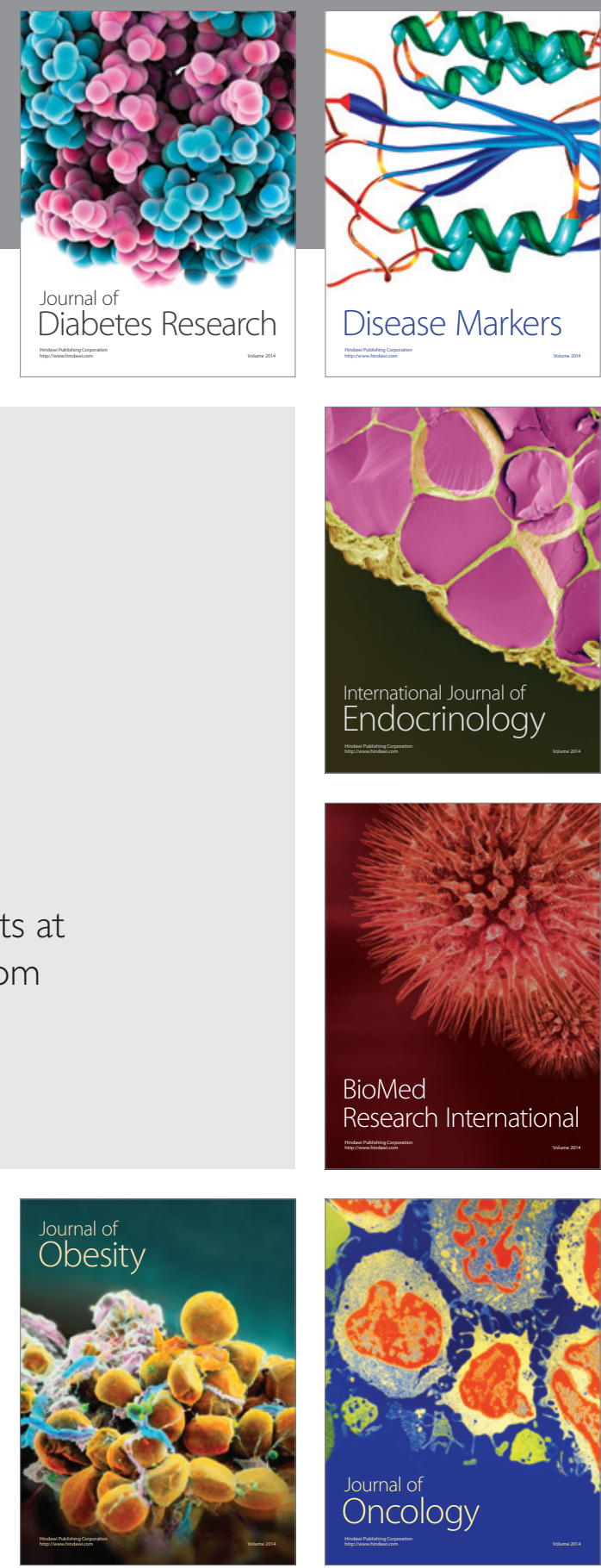

Disease Markers
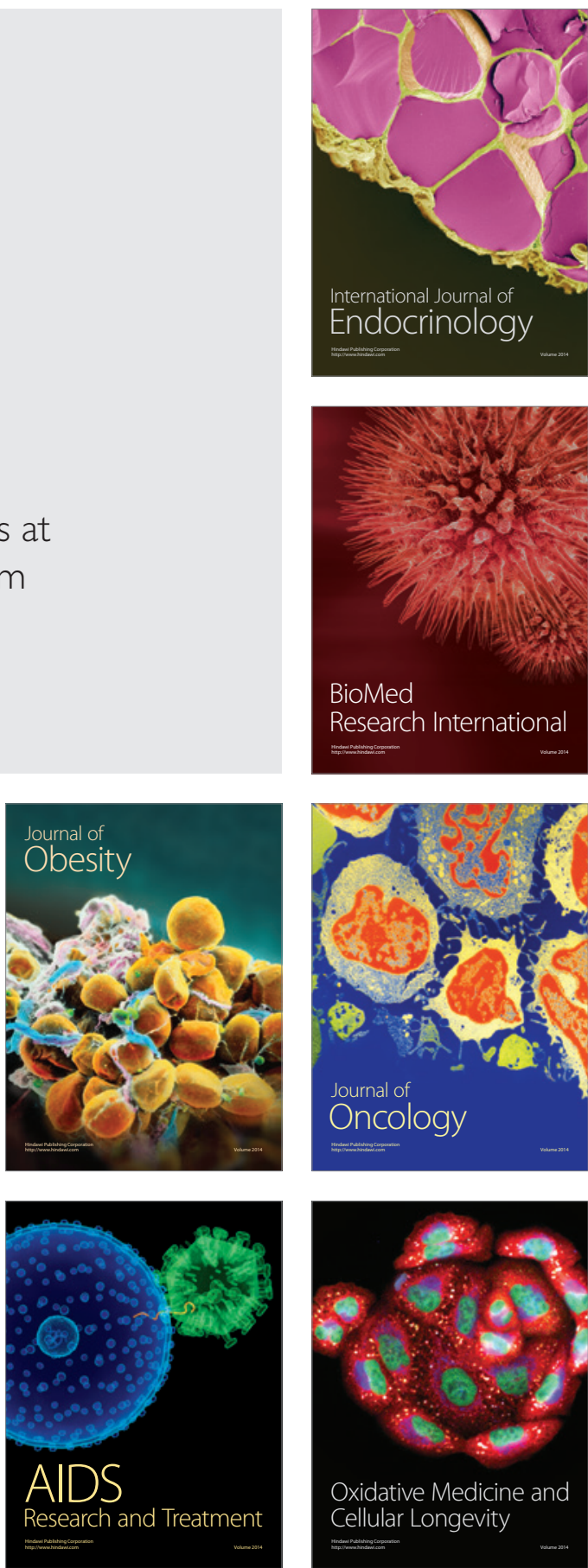